quite independent of the relationship between that affective state and other physiological processes and, in any case, depends upon a myriad of other biological and environmental factors. Furthermore, such a question could follow only from the underlying assumption that behaviourallyinduced alterations in immune function represent the single mechanism by which psychosocial factors influence disease. We know of no psychosomaticist or psychoimmunologist who would accept such a proposition and we cannot imagine how such a hypothesis could be derived from the available literature.

There are probably those in every field whose exuberance outdistances their data. One is not required to share their exuberance, but one is obliged to consider their data. One can only wonder, then, why anyone would want to throw out the baby with the bathwater. Connections between the CNS and the immune system are now being uncovered. Whatever forces were operating to set immunology apart, recent data suggest that much could be learned by studying immunoregulation as part of an integrated network of adaptive processes including behaviour. If not now, when?

ROBERT ADER

NICHOLAS COHEN

Departments of Psychiatry and

Microbiology (Immunology),

University of Rochester School of

Medicine and Dentistry,

Rochester, New York 14642, USA

1. Solomon, G.F. \& Amkraut, A.A. A. Rev. Microbiol. 35, 155-184 (1981)

2. Ader, R. Psychosom.. Med. 42, 307-321 (1980).

3. Maddox, J. Nature 309, 400 (1984)

4. Laudenslager, M.L., Ryan, S.M., Drugan, R.C., Hyson, R.L. \& Maier, S.F. Science 221, $568-570$ (1983)

5. Schleifer, S.J., Kellter, S.E. Camerino, M., Thornton, J.C. \& Stein, M. J. Am. med. Ass. 250, 374-377 (1983).

JOHN MADDOX WRITES - My article was a protest against those psychoimmunologists who attribute as yet unwarranted importance to the immune system (as distinct from other physiological systems) among the physiological correlates of behaviour. My statement that "people may be driven by adversity into decline is a familiar theme in literature" was not intended to demean psychoimmunology but, rather, to illustrate that the phenomena it seeks to account for have long been recognized.

My reference to heart attack and road accidents rather than, say, infection among the causes of death of those who have been psychologically shocked was intended to raise the precise question Ader and Cohen answered by saying that physiological systems other than the immune system may be involved.

I see nothing objectionable in the moderate statement of Ader and Cohen. Specifically, I can readily accept that there are many immunological consequences of, say, bereavement, although I do not believe that the tendency towards untimely death among bereaved spouses can be attributed primarily (or even at all) to the immune system (which is not to say that that may not be an attractive speculation).

Underlying this dispute (which seems to me not to be a substantial disagreement) is an important question. These reductionist days, it is proper to seek to identify the mechanisms by which states of mind cause physical illness. The immune system is one candidate, perhaps the most obvious after the adrenal hormone complex. But we all know that Freud's place in intellectual life rests in part on his careful documentation of the phenomena of psychosomatic illness, and on his explanation in terms of the unconscious, nowadays a somewhat oldfashioned concept.

So what if further investigation should show that the nervous system, and especially that part of it within the skull, plays an important part in the causation of psychosomatic illness? Such a development would no doubt be hailed as an important step towards Freud's rehabilitation, as a prophet if nothing more.

The point of this speculation, in the present context, is not to say that that is how it will turn out, but simply to suggest that the less obvious fields for explanation may ultimately be more productive. Much of the abuse that reductionists have recently attracted stems from their weakness for the kinds of explanations which happen to be at hand.

\section{An unexpected result in classical electrostatics}

SIR - A recent discussion of the structure of snowflakes (W.S. Mortley, Nature 313, 638 ; 1985) mentions the possible role of electric forces. In this and similar problems, establishing the least energy configuration appears vital. I would like to draw attention to a surprising result, which, to my best knowledge, has not been mentioned earlier.

What will be a minimum-energy configuration of a system of $N$ equal point charges $q$ placed inside a circle?

At first glance the answer is obvious: the Coulomb repulsion will arrange all $N$ charges at the vertices of a regular polygon inscribed into the circle (configuration $\mathrm{A}$ ). This statement seems to be so obvious that, apparently, nobody bothered to verify it by a direct calculation of the total electrostatic energy of the system $(W)$. However, such calculation, which can be easily performed on a programmable pocket calculator, shows, rather surprisingly, that this is true only for $N<12$. In units $q^{2} / R$ the total Coulomb energy $W$ is equal to 48.57568 for $N=11$ and 59.80736 for $N=12$. An alternative configuration (B), which is also compatible with the total symmetry of the system, has $N-1$ charges equally spaced along the circumference and one 'extra' charge at the centre of the circle. For $N=$ $11, W(\mathrm{~B})=48.62450$ (that is, greater than $W(\mathrm{~A})$ ), but for $N=12, W(\mathrm{~B})=59.57568$, that is, less than $W(\mathrm{~A})$. The non-equality $W(\mathrm{~B})<W(\mathrm{~A})$ also holds for $N>12$ (I verified this numerically to $N=400$, but very likely it is true for any $N>11$ )

The above result means that starting from $N=12$, the second configuration B (with one charge expelled to the centre of the circle) takes over from $\mathrm{A}$ as the minimum-energy configuration. In other words, it will be energetically beneficial for the system of $N$ equal point charges confined in a circle to 'expel' one charge to the centre of the circle (if $N>11$ ). Similar effect (the 'spontaneous ejection' of one charge to the geometrical centre) will also be likely to happen for $N$ charges inside the spherical surface $(N>$ ?). This may lead to some modification of usual theorems of electrostatic stability which claim that at the state of the equilibrium all charges in the conducting body are always located on the surface. The above example clearly illustrates that this is not necessarily so.

Alexander A. Berezin Department of Engineering Physics, McMaster University, Hamilton, Ontario, Canada L8S 4MI

\section{Spider pheromone 'packets'?}

SIR - The scanning electron micrograph (SEM) of the pustuliform organ of the spider Holcolaetis vidua Lessert (Salticidae) reproduced in Nature of 7 March (p.17) shows spherical bodies on the surface. It is suggested that the spheres may be 'packets' of pheromone secreted by the pores, on the assumption that the organ is pheromone producing ${ }^{1}$.

In reality the SEM shows that the spheres are not associated with the pores but occur on the pustuliform organs in proportion to the surface area occupied by the organs, about $25 \%$. The implied low volatility of the spheres seems inconsistent with pheromonal function. The mean diameter of the spheres of $1.3 \mu \mathrm{m}$, size distribution, aggregation and general appearance are consistent with the spheres coating the chorion of spiders' eggs of all families, including salticids, that I have examined ${ }^{2}$. These spheres may be found on the surface of spiders newly emerged from egg cocoons $^{2}$ and the brood guarding behaviour of salticids may expose the adults to such contamination.

\section{W.F. HUMPHREYS}

Western Australian Museum,

Francis Street,

Perth, Western Australia 6000

1. Wanless, F.R. 1984. Bull Br. Mus. (Nat. Hist.) Zoology series 47, 444-481.

. Humphreys, W.F. J. Zool. Lond. 200, 303-316 (1983).

\section{Scientific Corespondence}

Scientific Correspondence is intended to provide a forum in which readers may raise points of a rather technical character which are not provoked by articles or letters previously published (where Matters Arising remains appropriate). 